\title{
Sig2BioPAX: Java tool for converting flat files to BioPAX Level 3 format
}

\author{
Ryan L Webb, Avi Ma'ayan*
}

\begin{abstract}
Background: The World Wide Web plays a critical role in enabling molecular, cell, systems and computational biologists to exchange, search, visualize, integrate, and analyze experimental data. Such efforts can be further enhanced through the development of semantic web concepts. The semantic web idea is to enable machines to understand data through the development of protocol free data exchange formats such as Resource Description Framework (RDF) and the Web Ontology Language (OWL). These standards provide formal descriptors of objects, object properties and their relationships within a specific knowledge domain. However, the overhead of converting datasets typically stored in data tables such as Excel, text or PDF into RDF or OWL formats is not trivial for nonspecialists and as such produces a barrier to seamless data exchange between researchers, databases and analysis tools. This problem is particularly of importance in the field of network systems biology where biochemical interactions between genes and their protein products are abstracted to networks.

Results: For the purpose of converting biochemical interactions into the BioPAX format, which is the leading standard developed by the computational systems biology community, we developed an open-source command line tool that takes as input tabular data describing different types of molecular biochemical interactions. The tool converts such interactions into the BioPAX level $3 \mathrm{OWL}$ format. We used the tool to convert several existing and new mammalian networks of protein interactions, signalling pathways, and transcriptional regulatory networks into BioPAX. Some of these networks were deposited into PathwayCommons, a repository for consolidating and organizing biochemical networks.

Conclusions: The software tool Sig2BioPAX is a resource that enables experimental and computational systems biologists to contribute their identified networks and pathways of molecular interactions for integration and reuse with the rest of the research community.
\end{abstract}

\section{Background}

BioPAX is a protocol for the specification and representation of cell signaling pathways, gene-regulatory networks, protein-protein interactions and other types of biomolecular interaction data [1]. There are several software tools that use the BioPAX format for pathway visualization and analysis for hypotheses generation. For example, the popular tool Cytoscape allows customizable visualization and easy navigation of different types of networks [2]. Cytoscape plug-ins, including the popular BiNGO [3], and other plugins such as BiNoM [4], and cPath [5] further extend Cytoscape's capabilities for

\footnotetext{
* Correspondence: avi.maayan@mssm.edu Department of Pharmacology and Systems Therapeutics, Systems Biology Center New York (SBCNY), Mount Sinai School of Medicine, New York NY 10029, USA
}

(c) 2011 Webb and Ma'ayan; licensee BioMed Central Ltd. This is an Open Access article distributed under the terms of the Creative Commons Attribution License (http://creativecommons.org/licenses/by/2.0), which permits unrestricted use, distribution, and reproduction in any medium, provided the original work is properly cited. pathway analysis, data visualization, and data integration. BiNGO is a plugin that statistically analyzes a set of genes and their corresponding Gene Ontology functional annotations to determine which functional categories are overrepresented in that gene set. BiNGO uses Cytoscape's visualization capabilities to display the results. BiNoM is a plugin that performs structural analysis of networks, identifying strongly connected components, paths and cycles. cPath is an interaction database that can be included in Cytoscape as a plugin. The cPath database is a central repository for pathway and interaction datasets from multiple sources including MINT [6], IntAct [7], Reactome [8], and BioGRID [9]. The plugin allows for data retrieval from the central cPath database via an XML Web Services API, using the Cytoscape visualization engine for viewing biochemical 
networks. Interaction data stored in cPath are in BioPAX format.

BioPAX is one of several specification protocols that have been developed in an attempt to formally characterize biochemical regulatory molecular interactions. Some of these other specifications include the Proteomics Standard Initiative Molecular Interactions format (PSI-MI) [10] and the Systems Biology Markup Language (SBML) [11]. There are tools for conversion of some of these data formats into BioPAX. The previously mentioned Cytoscape plugin BiNoM also allows for conversion between BioPAX, SBML, and CellDesigner formats. However, most biochemical interaction data is not stored in one of these formats already, but rather stored in flat files, Excel spreadsheets, as network diagrams, or as tables in PDF format. While there are commercial products available to help researchers transform their flat text files into a general OWL format, to date, there are no tools available to transform flat files into BioPAX format. Such a tool would be useful because there are many pathway databases and networks that need to be converted for data sharing and reuse. Additionally, biologists that identify new interactions or describe new pathways in publications and do not have the technical expertise to convert their interaction data into BioPAX format will be able to do so with the tool.

\section{Implementation}

We created a Java-based tool, Sig2BioPAX, for the purpose of converting cell signaling pathways and interaction network datasets into BioPAX. The software package uses the Jena Library[12], which includes data structures for representation and modification of RDF and OWL files. In the implementation, BioPAX classes are represented as Java classes. The classes contain variables which store the settings and details necessary for proper instantiation of those classes. Program control is passed through a tree of functions which adds new instances of classes as necessary to represent the data from the input file. Reading the input flat text file lineby-line, Sig2BioPAX adds nodes to the OWL model sequentially. Before adding a new node, the program traverses the model to make sure that no identical node (that is, a node having the same children) already exists. Finally, the newly created model is written to an OWL file using the Jena library functions. See Figure 1 for a flow-diagram of the internals of the Sig2BioPAX program.

There are a finite number of reaction types that can be processed and converted into the BioPAX format. These reactions are described in Table 1. Sig2BioPAX mainly supports cell signaling reaction types including protein-protein binding interactions, protein kinase phosphorylations and phosphatase dephosphoryations,

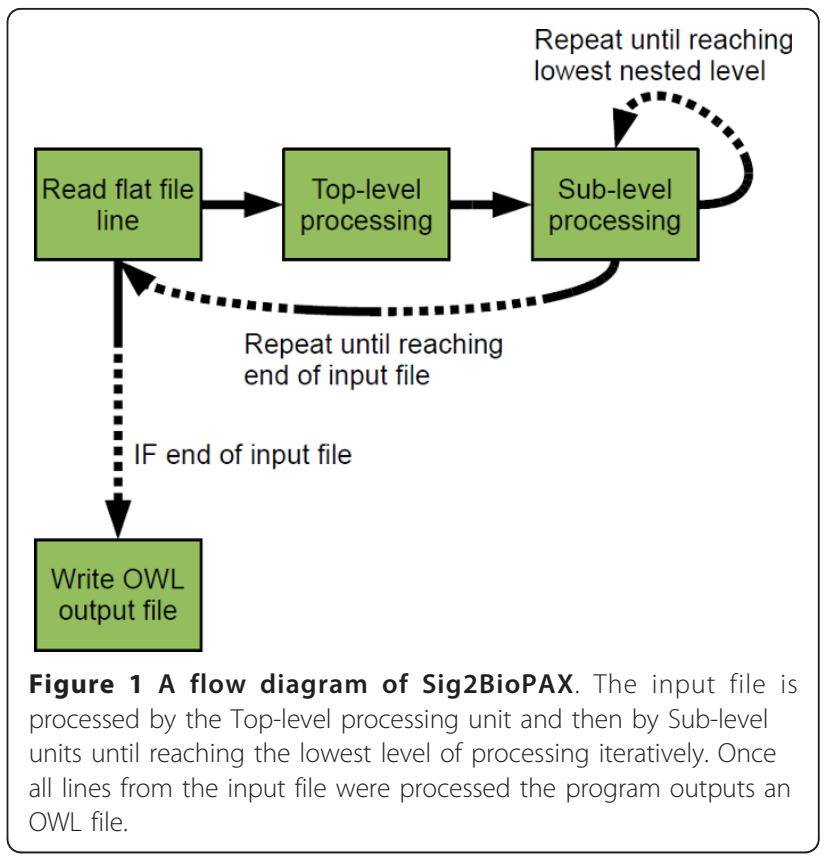

guanine nucleotide exchange reactions, and ubiquitinations. Since BioPAX level 3 has added support for transcriptional regulatory interactions, Sig2BioPAX can be used to convert protein/DNA interactions in the form of transcription-factors binding to regulatory regions of genes. Sig2BioPAX categorizes the input reaction lines based on user-supplied rules. These rules determine the logic used to convert the flat files to the BioPAX level 3 format. The rules must be specified in a rules file using a custom language which is fully described in the program's instruction manual and also available within the GUI version of the program by clicking the question mark button. A default rules.txt is included with the program's release. Users may modify the included rules. txt or create their own rules file from scratch.

One of Sig2BioPAX's accepted input parameter is the input file template type, which is a descriptor of the format of the input file. Four different input formats are accepted by the current version. The default type, sig, is described in Table 2. The second type, called source_target, forgoes describing the source and target types (i.e. kinase) and only describes the reaction type between the source and target (i.e. phosphorylation). The third template type is $t f_{-}$target, used to describe transcription factor binding to the promoter of target genes as determined by ChIP-seq or ChIP-chip types of experiments [13]. The fourth template type is sif, or simple interaction format, which is a format specifying the target and source names and the interaction type. In the implementation, input templates are stored within the Line.java source file, which contains the function ReadLine(). This function is composed of IF statements, one 
Table 1 A description of the reaction types processed by Sig2BioPAX

\begin{tabular}{ll}
\hline Reaction Type & Description \\
\hline Binding & Molecules bind to form a complex \\
Kinase Phosphorylation & Kinase catalyzes addition of phosphate group to target molecule \\
Dephosphorylation & Catalyst initiates removal of phosphate group from target molecule \\
Guanine Nucleotide Exchange & GDP removed from complex and replaced with GTP \\
GTPase activating protein & GTP bound to compound becomes GDP \\
Ubiquitination & Ubiquitin molecule is added to target compound \\
Deubiquitination & Ubiquitin molecule is removed from target compound \\
Sumoylation & Small ubiquitin-related modifier is attached to target molecule \\
Cleavage with Phospholipase C & PLC cleaves PIP2 into IP3 and DAG \\
Cleavage on Cysteine & Deactivating cleavage on a cysteine residue of target protein \\
Inactivating Cleavage & Deactivating cleavage of target molecule \\
Activating Cleavage & Cleavage of pro-protein into active form \\
Protein-protein Interaction & Otherwise unspecified reaction between two proteins \\
Transcription & Protein activates or inhibits transcription of gene products \\
Transcription Factor Promoter Binding & Transcription factor and protein bind to create complex \\
\hline
\end{tabular}

for each input template type. In this code file, the testing condition for the IF statement are defined. The input line is parsed through a series of statements. By modifying the order and content of these statements, the user can customize the input templates.

\section{Results}

Sig2BioPAX is packaged as an executable JAR file. The tool is available in both command line and graphical user interface (GUI) versions. The command line version of the program, accessible by using the command line argument -cmd, accepts input parameters including the input file name, output file name, rules file name, option to overwrite output file, and an input-template

\begin{tabular}{l} 
Table 2 A description of an input line using the default \\
input template, sig, and the meaning of the individual \\
elements on the input line \\
\hline sig template: SN SH SM ST SL TN TH TM TT TL E TI ID \\
\hline SN $\quad$ Source compound name \\
SH Source Swiss-Prot human accession number \\
SM Source Swiss-Prot mouse accession number \\
ST Source Type of compound \\
SL Source cellular location \\
TN Target compound name \\
TH Target Swiss-Prot human accession number \\
TM Target Swiss-Prot mouse accession number \\
TT Target Type of compound \\
TL Target cellular location \\
E Effect of source on target compound - Activating, inhibiting, or \\
TI Type of interaction \\
ID Pubmed ID of reference article
\end{tabular}

Each element must be separated by whitespace, and the line is terminated by the newline character. type. In the command line version of the program, these options are passed as command line arguments. For example, to use input file foo.txt, output file bar.owl, the rules file rulesfile.txt, and the overwrite option, the command is: 'java -jar sig2biopaxv4.jar - cmd -in:foo.txt -out:bar.owl -r:rulesfile.txt -o'. The sig input template type is the default input template type. In the GUI version of the program, the input, output, and rules files can be selected from a file browser and the other options can be set interactively (Figure 2). Sig2BioPAX can be downloaded from the Google Code project hosting site at http://code.google.com/p/sig2biopax/

We used Sig2BioPAX to convert several network datasets into the BioPAX format, and some of these networks are available on Pathway Commons [14], an international collaborative database of biomolecular pathways. The datasets we converted to BioPAX are original networks we extracted from the literature for the projects: the presynaptome [15], representing protein-protein interactions present in presynaptic nerve terminals of mammalian neurons, and the neuronal signalome [16], representing cell signaling interactions extracted from neuroscience literature describing combined cell signaling pathways in mammalian neurons, the adhesome [17], a network of interaction in focal adhesions; and a kinase-substrate network we constructed for the program KEA [18], kinase enrichment analysis, and ChEA [19], which stand for chip-seq/chip enrichment analysis.

\section{Conclusions}

As the bulk and complexity of genome-wide molecular data increases, methods for sharing and exchanging data need to be further developed. Effective standard representation of data enables seamless data exchange across platforms, tools and databases. However, converting existing 


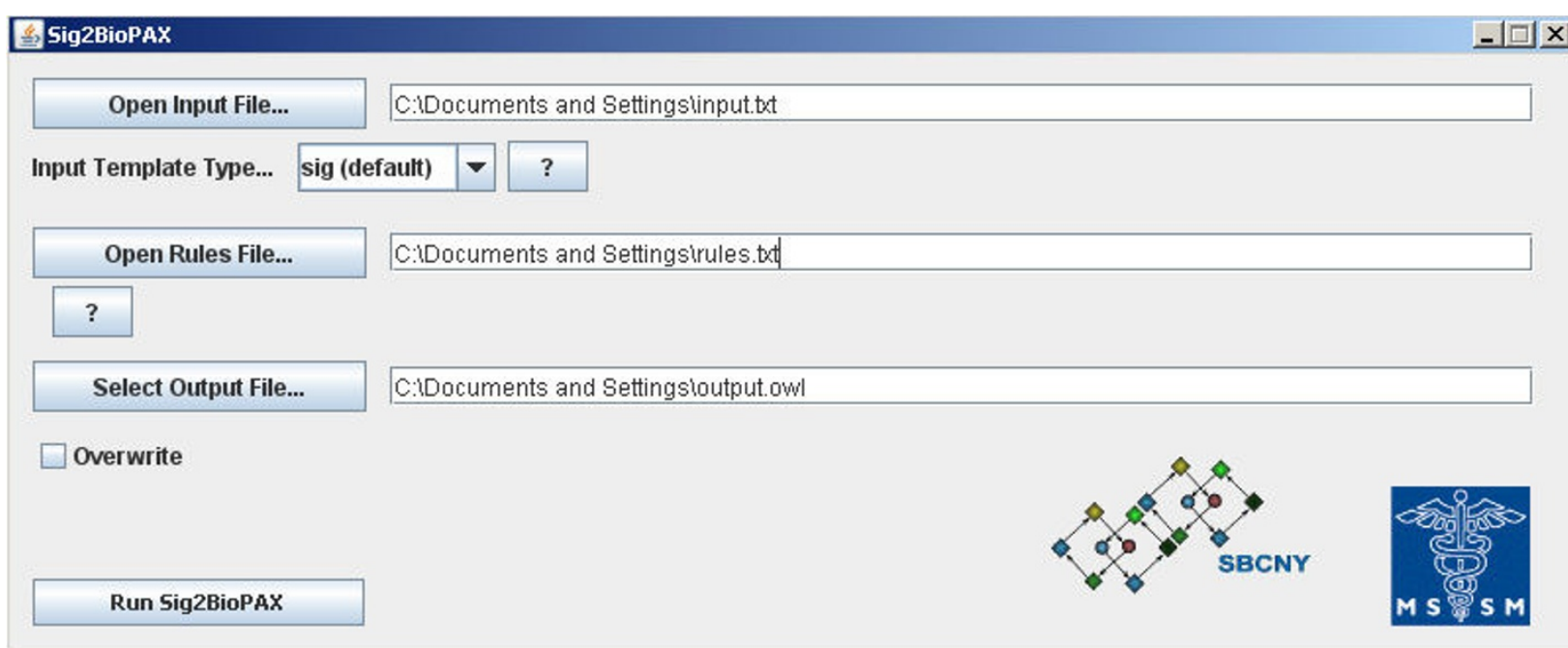

Figure 2 A screenshot of the GUI version of Sig2BioPAX. Users are provided with the ability to interactively specify the input file, the input file format, the rules file, and an output OWL file.

and new data into such exchange formats is not trivial. The Sig2BioPAX tool will further enable researchers to easily convert their flat file interaction data into the computable BioPAX format so that their data can be reused and interpreted by other researchers worldwide.

\section{Availability and Requirements \\ Project name: Sig2BioPAX}

Project home page: http://code.google.com/p/ sig2biopax/

Operating system(s): Platform independent

Programming language: Java

Other requirements: Jena 2.6.2 or higher

License: GNU GPL

Any restrictions to use by non-academics: none

\section{Acknowledgements}

This research was supported by NIH Grants P50GM071558-01A27398, R01DK088541, R01GM054508, R01DA15446, and KL2RR029885-0109. We would like to thank Emek Demir from MSKCC for assistance with the BioPAX specification

\section{Authors' contributions}

AM initiated and managed the project. RLW implemented and tested the Sig2BioPAX Java tool. AM and RLW wrote the manuscript. All authors read and approved the final manuscript.

\section{Competing interests}

The authors declare that they have no competing interests.

Received: 10 December 2010 Accepted: 21 March 2011 Published: 21 March 2011

\section{References}

1. Demir E, Cary MP, Paley S, Fukuda K, Lemer C, Vastrik I, Wu G, D'Eustachio P, Schaefer C, Luciano J, et al: The BioPAX community standard for pathway data sharing. Nat Biotechnol 2010, 28(9):935-942.
2. Cline MS, Smoot M, Cerami E, Kuchinsky A, Landys N, Workman C, Christmas R, Avila-Campilo I, Creech M, Gross B, et al: Integration of biological networks and gene expression data using Cytoscape. Nat Protoc 2007, 2(10):2366-2382.

3. Maere S, Heymans K, Kuiper M: BiNGO: a Cytoscape plugin to assess overrepresentation of Gene Ontology categories in Biological Networks. Bioinformatics 21(16):3448-3449.

4. Zinovyev A, Viara E, Calzone L, Barillot E: BiNoM: a Cytoscape plugin for manipulating and analyzing biological networks. Bioinformatics 2008, 24(6):876-877.

5. Cerami E, Bader G, Gross B, Sander C: cPath: open source software for collecting, storing, and querying biological pathways. BMC Bioinformatics 2006, 7(1):497.

6. Zanzoni A, Montecchi-Palazzi L, Quondam M, Ausiello G, HelmerCitterich M, Cesareni G: MINT: a Molecular INTeraction database. FEBS Letters 2002, 513(1):135-140.

7. Aranda B, Achuthan P, Alam-Faruque Y, Armean I, Bridge A, Derow C, Feuermann M, Ghanbarian AT, Kerrien S, Khadake J, et al: The IntAct molecular interaction database in 2010. Nucleic Acids Research 2010, 38(suppl 1):D525-D531.

8. Vastrik I, D'Eustachio P, Schmidt E, Joshi-Tope G, Gopinath G, Croft D, de Bono B, Gillespie M, Jassal B, Lewis $S$, et al: Reactome: a knowledge base of biologic pathways and processes. Genome Biology 2007, 8(3):R39.

9. Stark C, Breitkreutz BJ, Chatr-Aryamontri A, Boucher L, Oughtred R, Livstone MS, Nixon J, Van Auken K, Wang X, Shi X, et al: The BioGRID Interaction Database: 2011 update. Nucleic Acids Res 2010.

10. Hermjakob H, Montecchi-Palazzi L, Bader G, Wojcik J, Salwinski L, Ceol A, Moore S, Orchard S, Sarkans U, von Mering C, et al: The HUPO PSI's molecular interaction format-a community standard for the representation of protein interaction data. Nat Biotechnol 2004, 22(2):177-183.

11. Hucka M, Finney A, Sauro HM, Bolouri H, Doyle JC, Kitano H, Arkin AP, Bornstein BJ, Bray D, Cornish-Bowden A, et al: The systems biology markup language (SBML): a medium for representation and exchange of biochemical network models. Bioinformatics 2003, 19(4):524-531.

12. Jena - A Semantic Web Framework for Java. [http://jena.sourceforge.net/].

13. Lachmann A, Xu H, Krishnan J, Berger SI, Mazloom AR, Ma'ayan A: ChEA: transcription factor regulation inferred from integrating genome-wide ChIP-X experiments. Bioinformatics 2010, 26(19):2438-2444.

14. Cerami EG, Gross BE, Demir E, Rodchenkov I, Babur O, Anwar N, Schultz N, Bader GD, Sander C: Pathway Commons, a web resource for biological pathway data. Nucleic Acids Res 2010.

15. Abul-Husn NS, Bushlin I, Morón JA, Jenkins SL, Dolios G, Wang R, lyengar R, Ma'ayan A, Devi LA: Systems approach to explore components and interactions in the presynapse. PROTEOMICS 2009, 9(12):3303-3315. 
16. Ma'ayan A, Jenkins SL, Neves S, Hasseldine A, Grace E, Dubin-Thaler B, Eungdamrong NJ, Weng G, Ram PT, Rice JJ, et al: Formation of regulatory patterns during signal propagation in a Mammalian cellular network. Science 2005, 309(5737):1078-1083.

17. Zaidel-Bar R, Itzkovitz S, Ma'ayan A, lyengar R, Geiger B: Functional atlas of the integrin adhesome. Nat Cell Biol 2007, 9(8):858-867.

18. Lachmann A, Ma'ayan A: KEA: kinase enrichment analysis. Bioinformatics 2009, 25(5):684-686.

19. Lachmann A, Xu H, Krishnan J, Berger SI, Mazloom AR, Ma'ayan A: ChEA: transcription factor regulation inferred from integrating genome-wide ChIP-X experiments. Bioinformatics 26(19):2438-2444.

doi:10.1186/1751-0473-6-5

Cite this article as: Webb and Ma'ayan: Sig2BioPAX: Java tool for converting flat files to BioPAX Level 3 format. Source Code for Biology and Medicine 2011 6:5.

\section{Submit your next manuscript to BioMed Central} and take full advantage of:

- Convenient online submission

- Thorough peer review

- No space constraints or color figure charges

- Immediate publication on acceptance

- Inclusion in PubMed, CAS, Scopus and Google Scholar

- Research which is freely available for redistribution

Submit your manuscript at www.biomedcentral.com/submit 